\title{
COVID-19 and Its Impact on Cancer Patient's Outcome and Cancer Research
}

\author{
Nalin Goyal ${ }^{1}$, Deepak Saini ${ }^{2}$, Harpreet Angural', Richa ${ }^{1}$, Vikrant Kaushal', \\ Abhishek Shankar ${ }^{1}$
}

${ }^{1}$ Department of Radiation Oncology, Lady Hardinge Medical College and SSK Hospital, New Delhi, India. ${ }^{2}$ Division of Cancer Control \& Prevention, Indian Society of Clinical Oncology, Delhi, India.

\begin{abstract}
The current coronavirus pandemic has severely impacted the health care system in India and all around the world. All aspects of cancer care, including screening, investigations, treatment, clinical trials and research, have been affected as resources are diverted to combat the pandemic. Cancer patients are more susceptible to infections due to their immunocompromised status, either due to disease or therapy, and subsequently, this may result in in poor outcome, especially in case of coronavirus infection. Multiple studies have shown adverse impact of COVID-19 on cancer patients in form of higher mortality with haematological malignancy being worst affected, and interval between last oncological treatment and infection playing an important role in affecting the prognosis. Impact on clinical trials in oncology has been severe. Enrolment has decreased significantly, many trials have been suspended, and ongoing trials are facing problems with patient evaluations leading to impact on quality of data being generated. Telemedicine is now being used to overcome some of these problems but it has its own limitations and should be used judiciously. The pandemic has given us an opportunity to look into the shortcomings of our cancer care system and to find out ways to overcome them.
\end{abstract}

Keywords: Coronavirus- COVID-19- Telemedicine- Cancer Care- Cancer- Clinical Trials

Asian Pac J Cancer Care, 5 (Suppl 1), 199-201

Submission Date: 07/23/2020Ａcceptance Date: 08/11/2020

\section{Introduction}

The current coronavirus pandemic has severely impacted the health care system in India and all around the world. Among the worst affected are the cancer patients who are at higher risk of acquiring severe or critical form of infection and their survival is also impacted due to treatment breaks. Travel restrictions, imposition of lockdown and fear of infection on hospital visit for treatment are few reasons for treatment interruptions among cancer patients. Cancer patients are more susceptible to infections due to their immunocompromised status, either due to disease or therapy, and subsequently, this may result in in poor outcome, especially in case of coronavirus infection [1].

\section{Overall effects on cancer patient outcome}

In a cohort study by Kuderer et al. (2020) on clinical impact of COVID-19 on patients with cancer, the mortality was high in cancer patients $(13 \%)$ and was associated with risk factors like poor performance status, active disease and multiple comorbidities. Recent surgery or cytotoxic therapy was not found to be a risk factor for associated mortality, however morbidity was high with haematological malignancies, progressive disease status, poor performance status and recent surgeries [2].

In a study by Zhang et al. (2020), mortality was as high as $28.6 \%$ among cancer patients with COVID-19 infection and was more with patients who received anti-cancer treatment within last 14 days. Both of the above studies showed a higher mortality in cancer patients in comparison to $1.4 \%$ death rate seen in general population in a study by Liang et al. (2020) [3-4]. Another study showed a higher mortality rates seen in patients

\footnotetext{
Corresponding Author:

Dr. Nalin Goyal

Department of Radiation Oncology, Lady Hardinge Medical College and SSK Hospital, New Delhi, India.

Email: drnalingoyal@gmail.com
} 
with haematological malignancies (37\%) when compared with solid malignancies (25\%) [5] as taking care of hemato-oncology patients, is one of the most resource intensive of all medical therapy [6].

However, important factors such as patient demographics, cancer care centre facilities and the phase of pandemic should be taken into account while evaluating the effect on COVID-19 on cancer care. The outcome is also impacted by the ability of the patients to access treatment facilities which have restricted access in this crisis. This is either due to the travel restrictions which limits the ability of person residing in a rural area to reach a centre in urban area, or the operational restrictions at treatment centres.

\section{Effects on clinical trial and research}

Clinical trials in oncology have been severely impacted by this pandemic. According to a recent publication by Upadhaya et al. (2020), there was a significant impact on enrolment with only $1 / 5^{\text {th }}$ of institutions continuing enrolment [7]. Waterhouse et al. (2020) also showed, $54.8 \%$ decrease in patient visits owing to unwillingness or patient ability to present to hospital due to various reasons [8]. Patient care was the major concern along with staff safety. There was limitations in continuing radiology and laboratory services as important services were diverted for COVID-19 management. The trial deviations were also discussed by almost half of the investigators [8]. Decreased/missing patients visit impacted the data collection and will surely have a huge impact on the final result of trials. It will also adversely impact the patient outcome as missing interventions; be it radiotherapy, surgery or chemotherapy; will lead to upstaging of the disease. The quality of life will also be impacted as the health care facilities are burdened with COVID-19 leading to allocation of medical personnel and resources to take care of COVID -19 patients. Overall situation is reducing the access of cancer patients to both curative and palliative services. The gap in patient evaluation due to missed visits will lead to more investigations for assessment of disease at the time of next visit, thus leading to increase in total cost. The effect is more pronounced in studies directed to treatment for advanced cancer where survival is only for few months. Even a small gap in the treatment or follow up, would cause an anomaly in the collected data and may lead to negative results. There are concerns regarding the feasibility and continuity of ongoing trials from the investor side also as the returns on their investment in clinical trials will be impacted due to the treatment gaps and the resulting anomaly in the collected data. There have been reports regarding suspension of about 200 oncology trials in the month of March and April, reflecting the concerns regarding feasibility of oncology trials [7].

\section{Way Forward}

There is a need for alternate approach if we want, to continue with clinical services for cancer patients and to resume clinical research. Telemedicine is one of the alternative to avoid face to face consultations to prevent transmission of infection, but it will require broadband coverage for video consultations. It is helping cancer patients to minimise fear and anxiety but it is of not much use in overall management of cancer patients, especially the treatment aspect. There is a need to take adequate steps to ensure patient privacy and safety by imparting training for proper use of such facilities. There should be an arrangement for such consultation to nearby centres for an area with insufficient broadband coverage.

These modalities are working best for cancer patients who are on follow up. Challenges are more in India where cancer care centres are concentrated mainly in urban areas and most of the patients travel long distances for cancer treatment.

There have been discussions on home based chemotherapy. It may look feasible for some oral drugs, but most of the drugs administration need specialised care in dedicated cancer clinics or hospitals. Chemotherapy may have severe adverse effects, so it is not safe to administer at home. In addition, there are also logistics issues to make site visits by health care providers in view of restrictions imposed to tackle the pandemic.

This pandemic has highlighted the shortcomings of our system and gave us an insight to work positively in the direction of establishing more cancer centres, especially in the rural areas to minimise disparities and inequalities in access to cancer care. This will lead to a better access of patients with cancer to centres with multidisciplinary facilities closer to an area they reside. There is also a need to improve the preventive and screening facilities as this will help in reducing the incidence of cancer and overall burden on the health care system. These are some of the measures which might be useful for providing better cancer care.

\section{References}

1. Shankar A, Saini D, Bhandari R, Bharati SJ, Kumar S, Yadav G, Durga T, Goyal N. Lung cancer management challenges amidst COVID-19 pandemic: hope lives here. Lung Cancer Management. 2020 09;9(3):LMT33. https://doi.org/10.2217/ lmt-2020-0012

2. Kuderer NM, Choueiri TK, Shah DP, Shyr Y, Rubinstein SM, Rivera DR, et al. Clinical impact of COVID-19 on patients with cancer (CCC19): a cohort study. The Lancet. 2020 06;395(10241):1907-1918. https://doi.org/10.1016/ s0140-6736(20)31187-9

3. Zhang L, Zhu F, Xie L, Wang C, Wang J, Chen R, et al. Clinical characteristics of COVID-19-infected cancer patients: a retrospective case study in three hospitals within Wuhan, China. Annals of Oncology. 2020 07;31(7):894-901. https:// doi.org/10.1016/j.annonc.2020.03.296

4. Liang W, Guan W, Chen R, Wang W, Li J, Xu K, et al. Cancer patients in SARS-CoV-2 infection: a nationwide analysis in China. The Lancet Oncology. 2020 03;21(3):335-337. https://doi.org/10.1016/s1470-2045(20)30096-6

5. Mehta V, Goel S, Kabarriti R, Cole D, Goldfinger M, Acuna-Villaorduna A, et al. Case Fatality Rate of Cancer Patients with COVID-19 in a New York Hospital System. Cancer Discovery. 202005 01;10(7):935-941. https://doi. org/10.1158/2159-8290.cd-20-0516

6. Seth T, Shankar A, Roy S, Saini D. Hemato- Oncology Care in COVID-19 Pandemic: Crisis within a Crisis. Asian Pacific Journal of Cancer Prevention. 202005 01;21(5):1173-1175. 
https://doi.org/10.31557/apjcp.2020.21.5.1173

7. Upadhaya S, Yu JX, Oliva C, Hooton M, Hodge J, HubbardLucey VM. Impact of COVID-19 on oncology clinical trials. Nature Reviews Drug Discovery. 202005 18;19(6):376-377. https://doi.org/10.1038/d41573-020-00093-1

8. Waterhouse DM, Harvey RD, Hurley P, Levit LA, Kim ES, Klepin HD, et al. Early Impact of COVID-19 on the Conduct of Oncology Clinical Trials and Long-Term Opportunities for Transformation: Findings From an American Society of Clinical Oncology Survey. JCO Oncology Practice. 2020 07;16(7):417-421. https://doi.org/10.1200/op.20.00275

This work is licensed under a Creative Commons AttributionNon Commercial 4.0 International License. 